\title{
28 Research Square \\ Stability of Implants Supported By Three Types of Bone Grafting Materials in Dogs
}

\section{Mahdi Gholami}

Mashhad University of Medical Sciences

Farzaneh Ahrari ( $\nabla$ farzaneh.Ahrari@gmail.com )

Mashhad University of Medical Sciences https://orcid.org/0000-0003-1257-8881

Hamideh Salari Sedigh

Ferdowsi University of Mashhad

Christoph Bourauel

University Hospital Bonn: Universitatsklinikum Bonn

\section{Latifeh Ahmadi}

Mashhad University of Medical Sciences

\section{Research}

Keywords: Autografts, dental implant, osseointegration, resonance frequency analysis, synthetic bone graft, xenografts

Posted Date: November 15th, 2021

DOI: https://doi.org/10.21203/rs.3.rs-1063509/v1

License: (c) (i) This work is licensed under a Creative Commons Attribution 4.0 International License. Read Full License 


\section{Abstract}

Background: This study was conducted to assess the stability of implants placed in a simultaneous procedure with different grafting materials (autogenous, xenogenous, and synthetic) in experimentally induced bone defects in dogs.

Methods: Thirteen dogs were included and divided into three groups according to the time of sacrificing. Oversized osteotomies were prepared in the sternum, and the implants were placed in bone defects. A total of 3 to 5 implants were placed per animal. Each group of animals contained 3 subgroups according to the grafting material utilized. In subgroup 1, autograft was applied, whereas in subgroups 2 and 3 , bovine bone mineral (Cerabone) and a synthetic calcium phosphate substitute (Osteon II) were employed. At the end of the specified healing periods (2 months, 4 months, or 6 months), the animals were sacrificed and the implant stability was determined through measuring the resonance frequency.

Results: Forty-five integrated implants were obtained from this study and nine were lost (failure rate $17 \%$ ). The two-way analysis of variance revealed no significant difference in ISQ measurements either between the bone graft materials (autogenous, xenogenous, and synthetic; $P=0.950$ ) or between the healing intervals ( 2 months, 4 months, and 6 months; $P=0.769$ )

Conclusions: The stability of implants augmented with autogenous, xenogenous (Cerabone) or synthetic (Osteon II) graft materials was comparable at 2, 4 and 6 months after placement. This indicates that both Cerabone and Osteon II could be considered as suitable substitutes for regeneration of bone defects to overcome the limitations of autografts.

\section{Introduction}

Dental implants are considered as safe and ideal replacements for teeth lost due to various reasons and thus, the number of people who request this rehabilitation modality is growing incrementally. The failure rate of implants is influenced by several factors including the quantity and quality of recipient bone, the surface characteristics of the implants, the surgical technique, and the design of future restoration. The presence of an adequate bony support is a critical factor in implant survival. However, bone volume may be insufficient in cases that require immediate implant placement in large extraction sockets, or those lacking native bone support due to developmental anomalies, trauma or atrophic alveolar ridges.

Furthermore, the quality of bone may be inadequate in cases with oral infections or periodontal diseases. In these cases, restoring bone support with grafting materials is mandatory to provide optimal stability for endoessous implants.(1)

There are a variety of bone replacements to be employed for esthetic and regenerative applications in medicine and dentistry, including autogenous, allogenous, xenogenous, and synthetic (alloplastic) bone substitutes. These materials differ in the origin, expense, availability, and the associated morbidity. The autograft is harvested from the patient's skeleton and is considered the golden standard for bone reconstruction, as the live osteoblasts in the autograft provide osteoconductive, osteoinductive, and 
osteogenetic characteristics. Despite the mentioned benefits, autografts are associated with some disadvantages including the need for two surgical procedures (donor and recipient sites), which inevitably increases patient discomfort as well as the cost and time of operation. Furthermore, the utilization of autograft in large bony defects is limited by the quantity of bone that could be harvested from the donor site. $(2,3)$ The application of alternative graft materials has been suggested to overcome these limitations.

Xenografts are obtained from other species such as cows or horses and have osteoconductive potential. Cerabone (Botiss Biomaterials $\mathrm{GmbH}$, Zossen, Germany) is an inorganic osseous matrix that is manufactured from bovine cancellous bone by heating at the temperature above $1200^{\circ} \mathrm{C}$. This unique production process leads to complete elimination of organic components and albuminous impurities and thus minimizing the chance of disease transposition or immunogenic reactions.

The synthetic (alloplastic) grafts are mainly produced from calcium phosphate and exhibit osteoconductive properties.(4) The alloplastic materials would be available in an unlimited supply with no need to a donor site from the human or animal. Because of their synthetic nature, there is no risk of disease transmission, and their chemical similarity to human bone makes them biologically acceptable. Osteone II (Genoss, Seoul, Korea) is a synthetic graft material that is composed of a hydroxyapatite scaffold and beta tricalcium phosphate ( $\beta$-TCP) in a weight ratio of $30 \%$ and $70 \%$, respectively.

There are several methods to assess the degree of stability of implants in animal and clinical studies. The resonance frequency analysis (RFA), introduced by Meredith et al in 1990s, is a popular, quantitative, reproducible and non-aggressive index that can indicate the implant stability at the time of placement (primary stability) or over the course of osseointegration (secondary stability).(5-8) The most recently developed RFA instrument is wireless (Osstell Mentor, Integration Diagnostics AB, Göteborg, Sweden), where a metal rod (SmartPeg; Integration Diagnostics) is screwed to the implant. On the top of the peg, there is a small magnet which is excited by the magnetic pulses from the measurement probe. The frequency signal (according to $\mathrm{Hz}$ ) from the peg is achieved by the device and is indicated as the implant stability quotient (ISQ), from 1 to 100.

There are few human and animal experimental studies that compared the effect of different bone substitutes on the stability of implants placed in a concurrent procedure. $(1,2,9,10)$ Therefore, the present study was conducted to assess the stability of implants placed in a simultaneous procedure with different grafting materials (autograft, bovine bone graft and synthetic graft) in experimentally induced bone defects in dogs, through the resonance frequency analysis.

\section{Material And Methods}

\section{Animals}

The study was performed on 13 male Iranian dogs with 18 to 36 months age, and body weight of 20 to $35 \mathrm{Kg}$. Before experimentation, the animals underwent a 2-week quarantine period to ensure health and 
acclimation. The animals were kept in a special-designed room and fed with nutripet diet (Karaj, Iran) and fresh water ad libitum. The experimental protocol was reviewed and approved by the Animal Care and Use Committee at Mashhad University of Medical sciences and was coded as IR.mums.sd.REC.1394.130.

\section{The study design}

The dogs were numbered and divided into three groups according to the time of sacrificing. The study design consisted of preparing oversized osteotomies in the sternum (chest bone) of dogs, inserting implants in these bone defects, and filling the gap surrounding the implants by either of the three bone graft materials (autogenous, xenogenous or synthetic grafts). Each animal received all types of bone replacements. Five dogs were sacrificed after 2 months, whereas 4 at the 4-month and 4 at the 6-month intervals. The secondary stability of implants was measured and the samples were designated for biomechanical, $\mu$-CT and histology examinations. Only the result of mechanical testing is reported here.

\section{Medicaments}

Prior to surgery, intramuscular injection of Acepromazine 1\% (0.01-0.02 mg/Kg; Alfasan, Woden, Netherland) was administered and an angiocatheter was placed. Then, a combination of ketamine $10 \%$ (10 mg/kg; Alfasan) and diazepam 10 (10 mg/2ml; Caspian Tamin Co, Tehran, Iran) was intravenously infiltrated to induce general anesthesia. The dogs were intubated and anesthesia was maintained by inhalation of $2.5 \%$ to $3 \%$ isoflurane in 02 .

Antibiotic therapy was contemplated by slow intravenous injection of $1 \mathrm{~g}$ cefazolin (Loghman, Iran) before and after surgery. The administration of this medicament was continued through intramuscular injection every 12 hours for 7 days. The animals were also medicated with analgesics during operation using Meloxicam 2.0\% (0.2 ml/Kg body weight; Razak, Iran), and postoperatively (Meloxicam tablet 7.5 mg, 1 tablet every 24 hours for 2 days; Loghman Co, Iran)

\section{Osteotomy procedure}

The oversized osteotomies were prepared in the sternum (chest bone). Typically, the sternum of a dog is made up of seven segments (Fig 1) including a modified anterior segment (the pre-sternum), five narrow, cylindrical segments or sternebrae (forming the body of the sternum), and a uniform process (a hind segment).

Before operation, the surgical field was shaved and disinfected with $7.5 \%$ povidone-iodine solution. The incision started at the site of the second rib and continued until the fifth rib using a No.15 surgical blade. Hemostasis was achieved by an electrocutter to create minimal amount of bleeding. The cutaneous and subcutaneous tissues and muscles were separated, and the periosteum was reflected using a fine periosteal elevator.

Following the exposure of sternum, 3 to 5 defects were created in the sternebrae of each animal (Fig 2) using a trephine burr (internal diameter $4 \mathrm{~mm}$; dentium, Seoul, South Korea). The bur was applied three 
times to create defects measuring $10 \mathrm{~mm}$ in length, $4 \mathrm{~mm}$ in width, and $6 \mathrm{~mm}$ in depth.

\section{Implant and bone graft Placement}

Through a standard technique, the $2 \mathrm{~mm}$ end of each defect was drilled using the ICX surgical kit. In this way, only the apical $2 \mathrm{~mm}$ of the implant engaged the bone, representing the worst possible condition for the primary stability of the implant, and the extreme need to employ bone graft materials. After that, a pure titanium screw implant (ICX; Medentis Medical GmbH, Demau, Germany), $3.45 \mathrm{~mm}$ in diameter and $6.5 \mathrm{~mm}$ long, was placed at the center of the defect and the cover screw was inserted. A total of 3 to 5 implants were placed per animal, according to the surgical and anatomic conditions. The Medentis Company provided the implants for this research project. All surgical procedures were contemplated under aseptic conditions.

Each group of animals contained 3 subgroups, as explained at the following. In subgroup 1 (autograft), autogenous bone particulates (particles sized 1-2 mm) were placed around the dental implant. The bone particles were harvested during preparation of the osteotomies at the time of operation. In subgroup 2 (Xenograft), the gap around the implant was augmented with deproteinized bovine bone mineral (particles sized 0.5-1 mm; Cerabone; Botiss Biomaterials $\mathrm{GmbH}$, Zossen, Germany). In group 3 (synthetic graft) a synthetic biphasic calcium phosphate substitute (particles sized 0.5-1 mm; Osteon Il; Genoss, Seoul, Korea) was applied to stabilize the implant.

The first defect in each of the 2-month, 4-month, or 6-month groups of animals was filled with autografts, the second with xenograft, and the third with the synthetic graft. This order was continued, so that the 5 dogs that were designed to be euthanized after 2 months received 21 implants surrounded by three types of grafts ( $n=7$ per subgroup), whereas the dogs in the 4-month and 6-month groups received 18 implants surrounded by three bone graft materials ( $n=6$ per subgroup). All bone powders were hydrated with sterile saline before placement in the cavity.

After packing the bone graft, the excess was removed and a resorbable collagen membrane (Jason Membrane; Botiss Biomaterials $\mathrm{GmbH}$, Zossen, Germany) was employed to cover the augmented bone defect. The wound was closed in four layers (periosteum, muscle, subcutaneous tissue and skin) using an absorbable 2-0 vicryl suture (Supabon, Iran) for the first three layers and a non-absorbable suture 2-0 nylon suture (Supabon) for skin.

\section{Post-surgical procedures}

After surgery, the surgical field was covered by sterile gauze dressing. The dressing was changed daily for 7 days, and a cervical collar was applied to prevent animal from licking the incision area. The animals were kept in a warm place and the antibiotic and analgesic medicaments were administrated for postoperative infection and pain control, as explained previously. The animals were monitored by the veterinarian and animal care staff to detect any signs of infection. In 4 animals, the incision area showed 
dehiscence and was treated by repeated suturing and antibiotic therapy. After about 10 days, the sutures were removed in cases that showed favorable healing under sedation with diazepam and kethamine.

\section{Implant stabilization assessment}

At the end of the specified healing periods ( 2 months, 4 months, or 6 months), the animals were sedated and then sacrificed via intravenous injection of overdosed sodium pentobarbital. The sternum was surgically reopened and the stability of implants was determined through measuring the resonance frequency by an Osstell Mentor (Integration Diagnostics AB, Gothenburg, Sweden). For this purpose, the closed cover screw over the implant was removed and a magnetic peg (SmartPeg Type II, Integration Diagnostics AB, Göteborg, Sweden) was screwed into the implant. The plastic probe of the device, which contains a magnetic material in its upper part, was placed near the peg at four directions including up: toward the animal's head, bottom: toward the foot of the animal, and toward the two sides of the body. The resulting vibration was detected by the device as the implant stability quotient (ISQ), and the mean of the four measurements was considered as the final ISQ value for each implant.

After measuring the implant stability, the sternebrae containing the implant and surrounding bone was excised by a saw. The specimens were kept in $10 \%$ neutral buffered formalin and were sent to the Oral Technology Department at the University of Bonn, Bonn, Germany for further biomechanical, $\mu$-CT and histologic examinations. The results of further testing will be reported in future.

\section{Statistical analysis}

The normal distribution of the data was confirmed by the Shapiro-Wilk test $(P>0.05)$. A two-way analysis of variance was run to detect any significant difference in stability measurements between the different healing times and various bone graft materials. The data were analyzed through the SPSS 13.0 software (SPSS Inc, Chicago, II, USA) and the level of statistical significance was set at $P<0.05$.

\section{Results}

In this study, 57 implants were placed in 13 animals. One dog that was designed to be euthanized after 6 months was expired a few days before the completion of the healing period, and 3 inserted implants were lost and withdrawn from the study. Therefore, the number of implants in each subgroup of graft materials was $n=7$ in the group of animals sacrificed after 2 month, $n=6$ in the group of animals that were euthanized after 4 months, and $n=5$ in dogs that were sacrificed after 6 months. In total, 54 implants were assessed in this study.

After opening the sternum at the designated intervals ( 2 months, 4 months, 6 months), it was observed that 3 implants were explanted during the wound healing procedure and 6 implants showed extensive mobility. These cases were considered as failures and were not included in the statistical analysis (failure rate 17\%). Altogether, 45 integrated implants were obtained from this study and nine were lost.

\section{Resonance frequency assessment}


Table 1 presents the mean and standard deviation (SD) of ISQ values for the implants supported by three graft materials at different healing intervals. The autograft stabilized sites demonstrated the highest magnitude of ISQ at the 2-month time point among the graft materials, and exhibited a negligible increase at the 6-month time point. The bovine bone graft sites showed lower stability at the 2-month interval than both the autogenous and synthetic graft sites, but experienced a small and steady increase in stability over the period of the experiment. The synthetic graft sites showed some increase in ISQ value between the 2- and 4- month time points, but the ISQ value dropped to the initial level at the 6-month interval.

Table 1

The total number $(n)$, the number of integrated implants $(\mathrm{N})$, and the mean and standard deviation (SD) of ISQ values according to grafting materials and healing intervals

\begin{tabular}{|c|c|c|c|c|c|c|c|c|}
\hline Group & $2-n$ & nth $(n=21)$ & 4-n & onth $(n=18)$ & $6-m$ & nth $(n=15)$ & Tol & $(n=54)$ \\
\hline Subgroup & $\mathrm{N}$ & Mean \pm SD & $\mathrm{N}$ & Mean \pm SD & $\mathrm{N}$ & Mean \pm SD & $\mathrm{N}$ & Mean $\pm S D$ \\
\hline Authograft & 5 & $\begin{array}{l}65.12 \pm \\
12.41\end{array}$ & 4 & $\begin{array}{l}65.12 \pm \\
13.95\end{array}$ & 5 & $66.44 \pm 6.06$ & 14 & $\begin{array}{l}65.56 \pm \\
10.51\end{array}$ \\
\hline Cerrabone & 6 & $\begin{array}{l}63.25 \pm \\
10.13\end{array}$ & 4 & $\begin{array}{l}66.55 \pm \\
10.34\end{array}$ & 5 & $70.4 \pm 12.14$ & 15 & $\begin{array}{l}66.50 \pm \\
10.48\end{array}$ \\
\hline Osteone & 7 & $64.42 \pm 5.53$ & 5 & $68.2 \pm 10.46$ & 4 & $\begin{array}{l}64.07 \pm \\
12.28\end{array}$ & 16 & $\begin{array}{l}65.51 \pm \\
8.63\end{array}$ \\
\hline $\begin{array}{l}\text { Total } \\
\text { integrated }\end{array}$ & 18 & $64.26 \pm 8.81$ & 13 & $\begin{array}{l}66.62 \pm \\
10.94\end{array}$ & 14 & $66.97 \pm 9.87$ & 45 & $\begin{array}{l}65.85 \pm \\
9.67\end{array}$ \\
\hline
\end{tabular}

The two-way analysis of variance revealed no significant difference in ISQ measurements either between the various bone graft materials (autogenous, xenogenous, and synthetic; $\mathrm{P}=0.950$ ) or between the different healing intervals ( 2 months, 4 months, and 6 months; $P=0.769$ )

\section{Discussion}

The present study evaluated the stability of implants surrounded by three types of bone graft materials (autogenic, xenogenic and synthetic) in oversized osteotomies in dogs. The model simulated concurrent implantation and regeneration in too large defects where there is not enough bone contact to support the implant. The sternum was selected for implant placement, because the radiographic examination revealed that the dog mandible does not have enough space to accommodate multiple implants in such extensive defects. Furthermore, the use of sternum eliminated the effect of contamination by oral microbial flora and provided a more sterile condition for osseointegration. The structure of sternum resembles the upper jaw. The failure rate of implants was calculated as $17 \%$ in this study, which is higher than that reported in usual conditions (11-13). However, it has been demonstrated that the degree of implant failure is high in grafted bone and may even reach $25 \%$ in extensive defects.(14)

In the present study, a resorbable collagen membrane was utilized in order to cover the defect site after material placement, and to enhance guided bone regeneration (GBR) through preventing the invasion of 
undesirable soft-tissue cells and guiding the proliferation of osteogenic cells.(10) The collagen membrane is resorbable and has several properties such as easy manipulation, favorable effects on wound healing, low antigenicity, high mechanical strength, and no need to a second-stage surgery for removal. Despite these benefits, the collagen membranes show an unpredictable resorption time, which could influence the rate of bone formation.(3)

In the present study, the concurrent placement of implant and bone autograft resulted in ISQ values of $65.12 \pm 12.41,65.12 \pm 13.95$, and $66.44 \pm 6.06$ at intervals of 2,4 , and 6 months after operation. The presence of live cells in the autograft makes it a standard, safe and predictable option to restore bone defects. However, it may undergo quick resorption and its application would be limited when a large quantity of bone is required. $(2-4,9)$

The bovine bone mineral used in this study (Cerabone) demonstrated the mean ISQ value of $63.25 \pm$ 10.13 at 2 months, which increased to $66.55 \pm 10.34$ and $70.4 \pm 12.14$ at intervals of 4 , and 6 months after implantation. The ISQ values in the bovine bone graft group showed a small but steady increase over the healing period (about $11 \%$ up to the 6-month interval). Deproteinized bovine bone mineral has been considered a suitable alternative to autografts because of its great similarity to human bone structure. The interconnected pores and rough surface morphology of Cerabone facilitate the adhesion of proteins and bone forming cells and thus integration of particles occurs into newly formed bone. Bovine bone graft exhibits only superficial reosption and a high volume stability (15-17) to the extent that it is considered clinically nonresorbable.(18) Schlegel et al (18) utilized a bovine bone substitute or autogenous bone for sinus augmentation and reported the volume reduction of $16.5 \%$ in the bovine bone group and $39.8 \%$ in the autogenous group at 180 days.

The alloplastic material group (Osteon II) demonstrated the mean ISQ value of $64.42 \pm 5.53$ at 2 months. The ISQ values of implants augmented by Osteon II increased to $68.2 \pm 10.46$ at 4 months and then dropped down to $64.07 \pm 12.28$ at the 6 -month time point. Osteon II consists of a hydroxyapatite scaffold $(30 \%)$ and $\beta$-TCP $(70 \%)$ to overcome the limitations of each component. The hydroxyapatite provides volume stability and space maintenance, but it has low osteoconductivity. In contrast, $\beta$-TCP is more biodegradable and is rapidly remodeled and replaced by newly formed bone.

In the current study, the secondary stability of implants augmented by different grafting materials did not show a significant alteration over the course of healing. Although there was a small and steady increase in the stability of implants supported by Cerabone and some alterations in the stability of implants in the Osteon group, neither was statistically significant. These data indicate that the osseointegration of implants appears to be adequate after 2 months of concurrent implantation and grafting with either of the autograft, xenograft or alloplastic bone substitutes, and waiting until 6 months for loading the implant would not be associated with a significant benefit in enhancing the implant stability. Cannizzaro et al (19) also suggested that early loading (at about 7 weeks) could be applied for implants that exhibited a sufficient primary stability at the time of placement. 
The grafting materials utilized in this study (autograft, cerabone or Osteone) exhibited comparable efficacy in providing support for implants placed in large osteotomy defects, as measured by resonance frequency analysis. This means that the use of bovine bone graft or alloplastic bone substitute in conjunction with an implant would provide the same amount of stability as the use of autograft would, and these materials should be considered as ideal replacements for bone autografts. It seems that the degree of implant stability should not be considered a critical factor in choosing the type of grafting material to fill the gap in bony defects. Instead, other parameters such as availability, resorption time and stableness,(20) expense and biocompatibility may be focused on when choosing the bone substitute in the clinical applications.

The ISQ value at the time of placement or within 1 to 2 months later could be used as a valid tool to reveal the risk of future failure, $(21,22)$ but there is some controversy regarding the threshold value. Although the exact value has yet to be identified, Glauser et al.(22) revealed that ISQ values in the range of $49-58$ were associated with a failure risk of $18.2 \%$. Oh et al (5) believed that ISQ value less than 45 indicates a definite failure, whereas ISQ values in the range of 60 to 70 indicate implant success. It has been demonstrated that ISQ values below 44 and 54 were associated with failure rates of $100 \%$ and $9.5 \%$, respectively, whereas the risk of failure was 0 in implants showing ISQ > 54.(8) In contrast, Ersalani et al (23) believed that ISQ readings for successful integration should be defined specifically for each implant deign and at present there is no standardized range for the various systems. In this study, the mean ISQ obtained in all groups was greater than 60 , which was acceptable, but lower than that reported in some previous investigations. The relatively medium ISQ values obtained in this study could be related to the small sample size, and the presence of too large bony defects which were filled with grafting materials and thin and short implants. Another reason may be the concurrent regeneration and implant placement (immediate approach technique) which could jeopardize the treatment result.

The outcomes of this study are corroborated by previous studies that have reported a negligible difference in treatment results between various grafting materials utilized for dental applications. $(2,3,9$, 24) In a literature review, Friberg (24) indicated that the use of all types of bone graft materials produced satisfactory augmentation and allowed for placement of 7 to $10 \mathrm{~mm}$-long implants. In contrast to the outcomes of this study, some studies indicated the superiority of autogenous bone as compared to other grafting materials in implant dentistry. $(1,25)$ In some previous studies, the reported ISQ values were higher and the failure rates of implants were lower than those reported in the present investigation. (1113, 26-29) Bianconi et al (29) rehabilitated extensive postextraction defects in a single surgical session and reported $100 \%$ survival rate for both implants and grafts. The controversy between the results of this study and those of previous investigations may be related to several factors including the size of the bone defect, the size and surface characteristics of the implants, the one-stage versus two-staged surgical process, and the variations in the recipient sites of the implants.

The limitation of this study were the small number of animals, and the expiration of one dog before completing the healing process. Other limitations of the study were placing the implants in sternum rather than the maxilla or mandible and not loading the implants over the experiment. The resonance frequency 
was not measured at the time of implantation, because multiple implants were placed in large bony defects in sternum and the surgical procedure itself was too long and complicated. However, it has been demonstrated that implants exhibit a slight decrease in stability after installation, with a reversal at 3 to 4 week interval, so that he ISQ value is generally restored to the initial level at 8 weeks post-operation.(26)

Future studies should benefit from differing protocols and larger sample sizes to compare the stability and success rate of implants augmented with various grafting materials. Further randomized clinical trials are also warranted to provide an evidence-based insight regarding the stability of implants inserted with a concurrent grafting procedure in maxillary or mandibular bone, from the time of insertion over long time after implant loading.

\section{Conclusions}

Within the limitations of the present animal study:

1. The stability of implants augmented with autogenous, xenogenous (Cerabone) or synthetic (Osteon II) graft materials was comparable over the period of the experiment. This indicates that both Cerabone and Osteon II could be considered as suitable substitutes for regeneration of bone defects to overcome the limitations of autografts.

2. The concurrent implantation and grafting procedure resulted in comparable stability at intervals of 2,4 and 6 months after placement, using either of the grafting materials. Therefore, osseointegration of implants appears to be adequate after 2 months of simultaneous implantation and grafting process, and waiting until 6 months for loading the implant would not be associated with a significant benefit in enhancing the implant stability.

\section{Declarations}

Ethics approval: The experimental protocol was reviewed and approved by the Animal Care and Use Committee at Mashhad University of Medical sciences and was coded as IR.mums.sd.REC.1394.130.

Consent for publication: Not applicable

Availability of data and materials: The datasets used and/or analysed during the current study are available from the corresponding author on reasonable request.

Competing interests: The authors declare that they have no competing interests.

Funding: A grant from the vice-chancellor for research of Mashhad University of Medical Sciences

\section{Author's contributions:}

Mahdi Gholami: project development, supervision, manuscript editing 
Latifeh Ahmadi: data collection, performing animal experiment experiment, data analysis, manuscript writing

Hamideh Salari Sedigh: performing animal experiments, manuscript editing

Christoph Bourauel: project development, supervision, manuscript editing

Farzaneh Ahrari: data collection, data analysis, manuscript writing and editing

Acknowledgements: The authors would like to thank the vice-chancellor for research of Mashhad University of Medical Sciences for the financial support of this project (grant number 941412). The implant materials were kindly provided by the ICX Company. The results presented in this work have been taken from a student thesis (thesis number 2953).

\section{References}

1. Cohen DJ, Scott KM, Kulkarni AN, Wayne JS, Boyan BD. Acellular mineralized allogenic block bone graft does not remodel during the 10 weeks following concurrent implant placement in a rabbit femoral model. 2020;31:37-48.

2. Ribeiro M, Fraguas EH, Brito KIC, Kim YJ, Pallos D, Sendyk WR. Bone autografts \& allografts placed simultaneously with dental implants in rabbits. J Craniomaxillofac Surg 2018;46:142-47.

3. Paknejad M, Rokn A, Rouzmeh N, Heidari M, Titidej A, Kharazifard MJ, et al. Histologic evaluation of bone healing capacity following application of inorganic bovine bone and a new allograft material in rabbit calvaria. J Dent (Tehran) 2015;12:31-8.

4. Miron RJ, Sculean A, Shuang Y, Bosshardt DD, Gruber R, Buser D, et al. Osteoinductive potential of a novel biphasic calcium phosphate bone graft in comparison with autographs, xenografts, and DFDBA. Clin Oral Implants Res 2016;27:668-75.

5. Oh JS, Kim SG, Lim SC, Ong JL. A comparative study of two noninvasive techniques to evaluate implant stability: Periotest and Osstell Mentor. Oral Surg Oral Med Oral Pathol Oral Radiol Endod 2009;107:513-8.

6. Makary C, Rebaudi A, Sammartino G, Naaman N. Implant primary stability determined by resonance frequency analysis: correlation with insertion torque, histologic bone volume, and torsional stability at 6 weeks. Implant Dent 2012;21:474-80.

7. Meredith N, Shagaldi F, Alleyne D, Sennerby L, Cawley P. The application of resonance frequency measurements to study the stability of titanium implants during healing in the rabbit tibia. Clin Oral Implants Res 1997;8:234-43.

8. Sennerby L, Meredith N. Implant stability measurements using resonance frequency analysis: biological and biomechanical aspects and clinical implications. Periodontol 2000 2008;47:51-66.

9. Park YH, Choi SH, Cho KS, Lee JS. Dimensional alterations following vertical ridge augmentation using collagen membrane and three types of bone grafting materials: A retrospective observational 
study. Clin Implant Dent Relat Res 2017;19:742-49.

10. Soni R, Priya A, Agrawal R, Bhatnagar A, Kumar L. Evaluation of efficacy of platelet-rich fibrin membrane and bone graft in coverage of immediate dental implant in esthetic zone: An in vivo study. J Clin Periodontol 2020;11:67-75.

11. French $D$, Ofec R, Levin L. Long term clinical performance of 10871 dental implants with up to 22 years of follow-up: A cohort study in 4247 patients. 2021.

12. Moy PK, Medina D, Shetty V, Aghaloo TL. Dental implant failure rates and associated risk factors. International Journal of Oral \& Maxillofacial Implants 2005;20.

13. Motamedian SR, Khojaste M, Khojasteh A. Success rate of implants placed in autogenous bone blocks versus allogenic bone blocks: A systematic literature review. Ann Maxillofac Surg 2016;6:7890.

14. Lekholm U, Wannfors K, Isaksson S, Adielsson B. Oral implants in combination with bone grafts: a 3year retrospective multicenter study using the Brånemark implant system. International journal of oral and maxillofacial surgery 1999;28:181-87.

15. Gultekin BA, Cansiz E, Borahan O, Mangano C, Kolerman R, Mijiritsky E, et al. Evaluation of Volumetric Changes of Augmented Maxillary Sinus With Different Bone Grafting Biomaterials. J Craniofac Surg 2016;27:e144-8.

16. Jensen T, Schou S, Svendsen PA, Forman JL, Gundersen HJ, Terheyden H, et al. Volumetric changes of the graft after maxillary sinus floor augmentation with Bio-Oss and autogenous bone in different ratios: a radiographic study in minipigs. Clin Oral Implants Res 2012;23:902-10.

17. Mordenfeld A, Hallman M, Johansson CB, Albrektsson T. Histological and histomorphometrical analyses of biopsies harvested 11 years after maxillary sinus floor augmentation with deproteinized bovine and autogenous bone. Clin Oral Implants Res 2010;21:961-70.

18. Schlegel KA, Fichtner G, Schultze-Mosgau S, Wiltfang J. Histologic findings in sinus augmentation with autogenous bone chips versus a bovine bone substitute. Int J Oral Maxillofac Implants 2003;18:53-8.

19. Cannizzaro G, Felice P, Leone M, Viola P, Esposito M. Early loading of implants in the atrophic posterior maxilla: lateral sinus lift with autogenous bone and Bio-Oss versus crestal mini sinus lift and 8-mm hydroxyapatite-coated implants. A randomised controlled clinical trial. Eur J Oral Implantol 2009;2:25-38.

20. Heinemann F, Hasan I, Bourauel C, Biffar R, Mundt T. Bone stability around dental implants: Treatment related factors. Ann Anat 2015;199:3-8.

21. Sjöström M, Sennerby L, Nilson H, Lundgren S. Reconstruction of the atrophic edentulous maxilla with free iliac crest grafts and implants: a 3-year report of a prospective clinical study. Clin Implant Dent Relat Res 2007;9:46-59.

22. Glauser R, Sennerby L, Meredith N, Rée A, Lundgren A, Gottlow J, et al. Resonance frequency analysis of implants subjected to immediate or early functional occlusal loading. Successful vs. failing implants. Clin Oral Implants Res 2004;15:428-34. 
23. Ersanli S, Karabuda C, Beck F, Leblebicioglu B. Resonance frequency analysis of one-stage dental implant stability during the osseointegration period. J Periodontol 2005;76:1066-71.

24. Friberg B. Bone augmentation for single tooth implants: A review of the literature. Eur J Oral Implantol 2016;9 Suppl 1:S123-34.

25. Noelken R, Pausch T. Peri-implant defect grafting with autogenous bone or bone graft material in immediate implant placement in molar extraction sites-1- to 3-year results of a prospective randomized study. 2020;31:1138-48.

26. Han J, Lulic M, Lang NP. Factors influencing resonance frequency analysis assessed by Osstell mentor during implant tissue integration: II. Implant surface modifications and implant diameter. Clin Oral Implants Res 2010;21:605-11.

27. Ozkan Y, Ozcan M, Varol A, Akoglu B, Ucankale M, Basa S. Resonance frequency analysis assessment of implant stability in labial onlay grafted posterior mandibles: a pilot clinical study. Int J Oral Maxillofac Implants 2007;22:235-42.

28. Jensen SS, Aaboe M, Janner SF, Saulacic N, Bornstein MM, Bosshardt DD, et al. Influence of particle size of deproteinized bovine bone mineral on new bone formation and implant stability after simultaneous sinus floor elevation: a histomorphometric study in minipigs. Clin Oral Implants Res 2015;17:274-85.

29. Bianconi S, Bozzoli P, Del Fabbro M. Treatment of Postextraction Sites With Allograft-Stabilized Dental Implants: A Clinical Case Series. Implant Dent 2017;26:37-45.

\section{Figures}




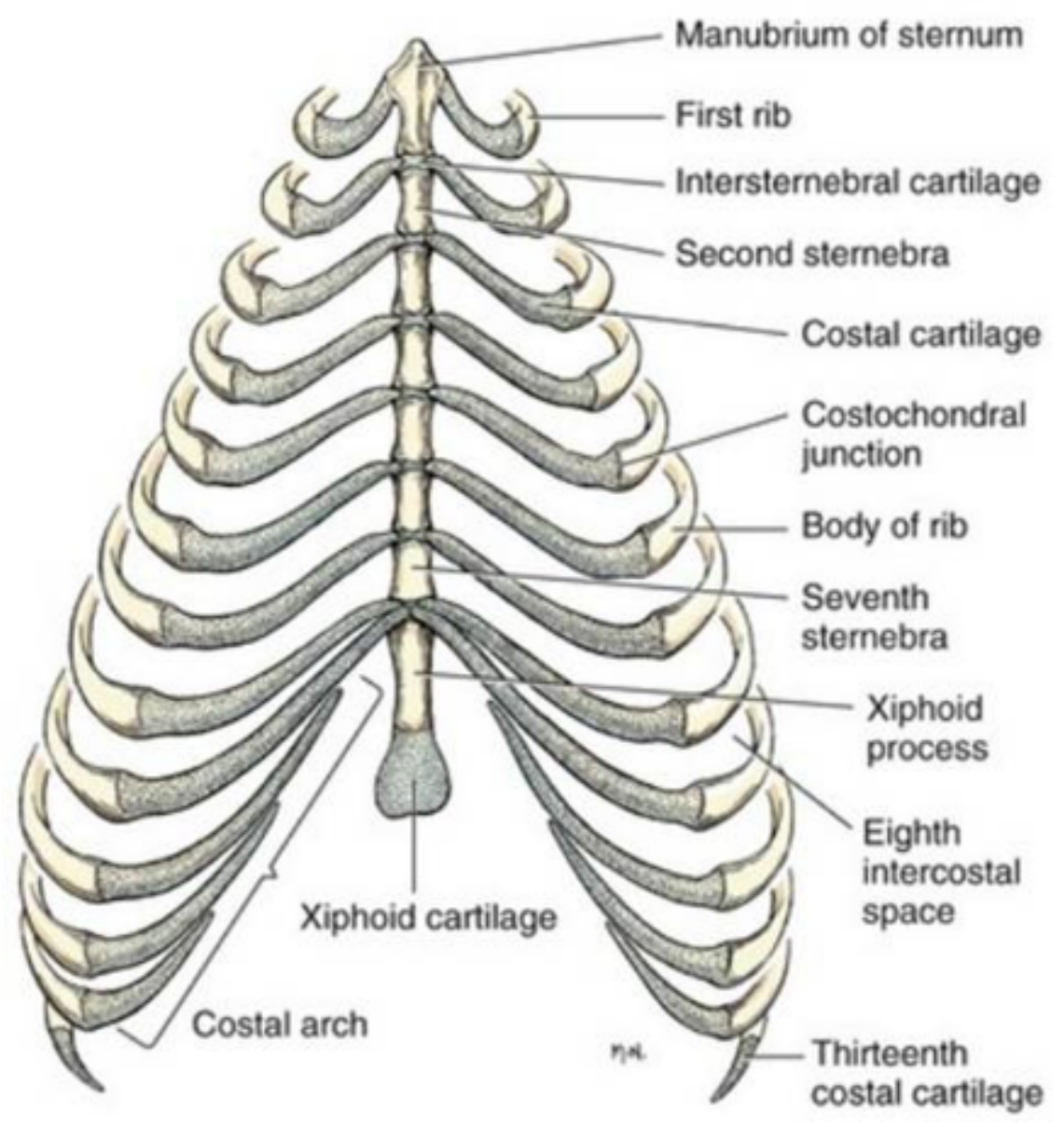

Figure 1

A schematic representation of a dog sternum 


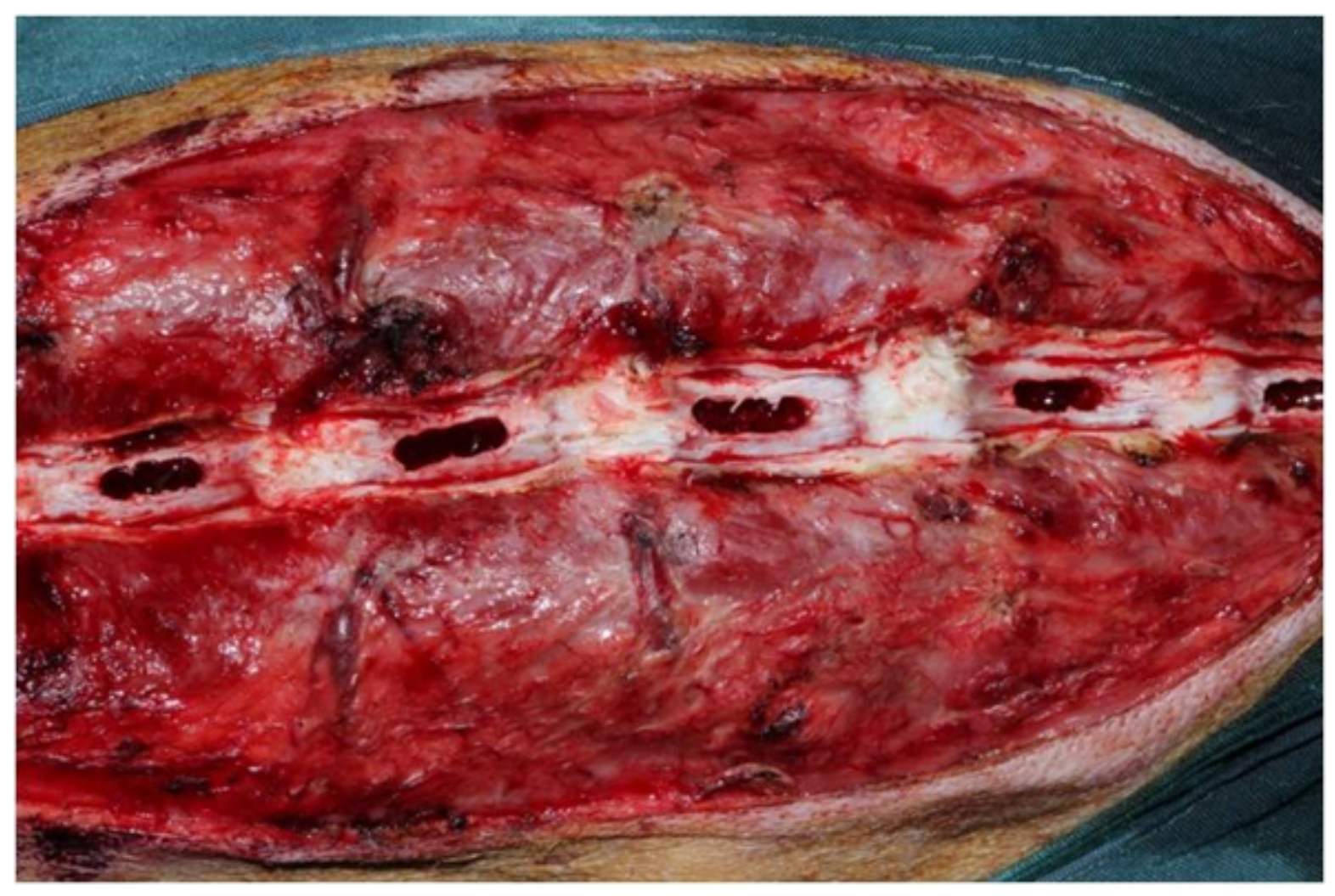

\section{Figure 2}

Preparing five oversized osteotomies for implant placement in the sternum 